\title{
Spatial patterns from a dispersal limitation perspective: revealing biotic interactions
}

Michael Kalyuzhny ${ }^{*}$ (michael.kalyuzhny@utexas.edu), Jeffrey K. Lake² (jlake@adrian.edu), Annette M. Ostling ${ }^{1}$ (annette.ostling@austin.utexas.edu)

1. Department of Integrative Biology, The University of Texas at Austin, 2415 Speedway, Austin, TX 78712, USA

2. Department of Biology, Adrian College, Adrian, MI, USA

Keywords: Conspecific Negative Density Dependence (CNDD), Heterospecific Negative Density Dependence (HNDD), coexistence, tropical forests, Janzen-Connell, Excess nearest neighbor Distance, Excess neighborhood abundance, Clark-Evans, spatial statistics, null models 


\section{Abstract}

Local spatial distributions of populations are often studied in comparison to Complete Spatial Randomness (CSR) and are found to be ubiquitously aggregated, likely due to dispersal limitation. Here we theoretically examine the advantages of comparing observed distributions to simulated populations subject only to drift and Dispersal Limitation (DL). Compared to this DL null, local competition produces overdispersion out to surprisingly large scales - much larger than the scale of competitive interactions. Furthermore, strong overdispersion provides a hallmark that a key requirement of stable coexistence is met, as it can only be observed if intraspecific competition is substantially stronger than interspecific competition. Dispersion compared to CSR is insensitive to competition and as a result unreflective of its presence. Hence, we suggest DL as a complement to CSR since the former focuses on biologically relevant spatial scales and has the potential to detect biotic interactions and habitat specificity. 


\section{Introduction}

The spatial distributions of species are considered among their most fundamental properties, begging for mechanistic explanation. Furthermore, most of the processes that shape ecological communities and their diversity and composition operate in a spatial context and leave a spatial mark. Consequently, spatial distributions contain information on the processes that created them and can be used to study these processes if analyses are constructed to properly disentangle them (McIntire \& Fajardo 2009; Wiegand \& Moloney 2013).

Here we focus on single species distributions at small scales, where individuals are often conceptualized as points in 2D space and the population distribution as a set of points or “(univariate) point pattern" (Wiegand \& Moloney 2013). The most basic perspective on such patterns entails drawing a fundamental division between cases when points are close to each other or dense (aggregated), and cases when they are far or sparse (overdispersed).

"Close/dense" and "far/sparse" are considered with respect to Complete Spatial Randomness (CSR) or Poisson null model, where points appear randomly, homogenously, and independently throughout the landscape (a random distribution, Wiegand \& Moloney 2013). Mechanistically, it has been argued that intraspecific aggregation can be caused by dispersal limitation (Chave et al. 2002; Svenning \& Skov 2002; Law et al. 2003); a spatially clumped habitat (Wiegand et al. 2007; Bagchi et al. 2011; Brown et al. 2016); and by positive interactions (Molofsky et al. 2001). Overdispersion, on the other hand, is primarily the result of negative interactions between individuals creating Conspecific Negative Density Dependence (CNDD, Chave et al. 2002; Law et al. 2003; Begon \& Townsend 2020).

Despite its promise, linking spatial patterns and processes proves challenging. First, a given spatial pattern could arise from several different processes (McIntire \& Fajardo 2009; Wiegand \& Moloney 2013), making it difficult to distinguish between them using the observed pattern alone. Moreover, in forests, the distribution of the vast majority of tree species proves to be aggregated (Pielou 1962; Hubbell 1979; Plotkin et al. 2000; Seidler \& Plotkin 2006) often at all measured spatial scales (Condit et al. 2000). This limits the usefulness of the fundamental division between 'aggregation' and 'overdispersion', since the latter is very rarely observed, and complicates mechanistic interpretation since aggregation has many possible causes.

The ubiquitous spatial aggregation of adult trees also contradicts the widespread evidence for strong CNDD acting on juveniles (Comita et al. 2010; Mangan et al. 2010; Johnson et al. 2012; Lebrija-Trejos et al. 2016), which should generate overdispersion. This evidence for CNDD was obtained by looking at the demography of single life stages (e.g., survival of firstyear seedlings) in response to conspecific density. However, CNDD at one life stage could be counteracted by processes at other life stages (Detto et al. 2019; Hülsmann et al. 2020). Moreover, much of the aforementioned evidence for CNDD potentially suffers from statistical biases (Detto et al. 2019). Analyzing the spatial distributions of adults could complement the demographic approach and potentially evaluate the overall cumulative effects of CNDD on the distribution and dynamics of adults. Such effects, however, are rarely found, as there is currently very limited evidence that the effects of CNDD propagate to adults (Mittelbach \& McGill 2019), 
suggesting that CNDD is overwhelmed, diluted, or obscured by the forces creating aggregation (as predicted by Chave et al. 2002; Brown et al. 2016). Since CNDD and other effects of biotic interactions are of primary importance for population and community dynamics (Hülsmann et al. 2020), it is very important to distinguish between the aforementioned scenarios: Does CNDD play no substantial role in the dynamics, or alternatively, do the effects of CNDD on adult distributions need to be teased apart from a backdrop of aggression?

Several approaches were developed for addressing this question. Already Pielou (1962) suggested truncating the distribution of nearest neighbor distances as a solution. In recent years, it was proposed to compare the observed pattern of individuals to a heterogenous Poisson null model, where different parts of the landscape have different expected densities (Wiegand et al. 2007). These methods require deciding how to "smooth" the density of individuals or to truncate the distribution of nearest neighbor distances. Furthermore, the mechanistic interpretation of the results is not always clear, since it is not evident what processes were "removed" by the method (Roughgarden 1983). Other works used the locations of juveniles as a null for the expected locations of adults (Condit et al. 2000; Bagchi et al. 2011), but these works found at most limited evidence for conspecific effects driving adult distribution patterns.

While multiple mechanisms shape community dynamics and spatial patterns, species must first disperse to a location, and only then they can interact with the a-biotic and biotic environment (“dispersal filtering”, e.g. Kraft et al. 2015; Cadotte \& Tucker 2017). Indeed, models of dispersal assembly, where species disperse and go extinct at random, are often used as null models for community dynamics and as a baseline for detecting biotic and a-biotic interactions (Hubbell 2001; Gotelli \& Ulrich 2012; Kalyuzhny et al. 2022). However, despite this "preemption" of dispersal, and despite considerable evidence for the importance of dispersal limitation in multiple systems (Hubbell et al. 1999; Muller-Landau et al. 2008; Losos \& Ricklefs 2010), the CSR null model typically used for analyzing spatial patterns (as well as its cluster extensions, Plotkin et al. 2000; Wiegand et al. 2009), distributes the randomized individuals all across the survey plot - a scale that has no biological relevance. For an uncommon species with limited dispersal, this null may distribute most individuals to many parts of the landscape inaccessible due to dispersal limitation, and in comparison, the observed pattern will seem strongly aggregated. For example, the hypothetical population in Figure 1a, subject to both dispersal limitation and CNDD, is aggregated compared to CSR (Figure 1b). However, it may be considered overdispersed (having fewer neighbors and longer distances to them, Figure 1d-e) compared to a null with only dispersal limitation (Figure 1c). Hence, the CSR null model may create unrealistic and mechanistically unjustifiable low density and long distances to neighbors, potentially obscuring the short-distance repulsion effects of CNDD and masking habitat specificity. 

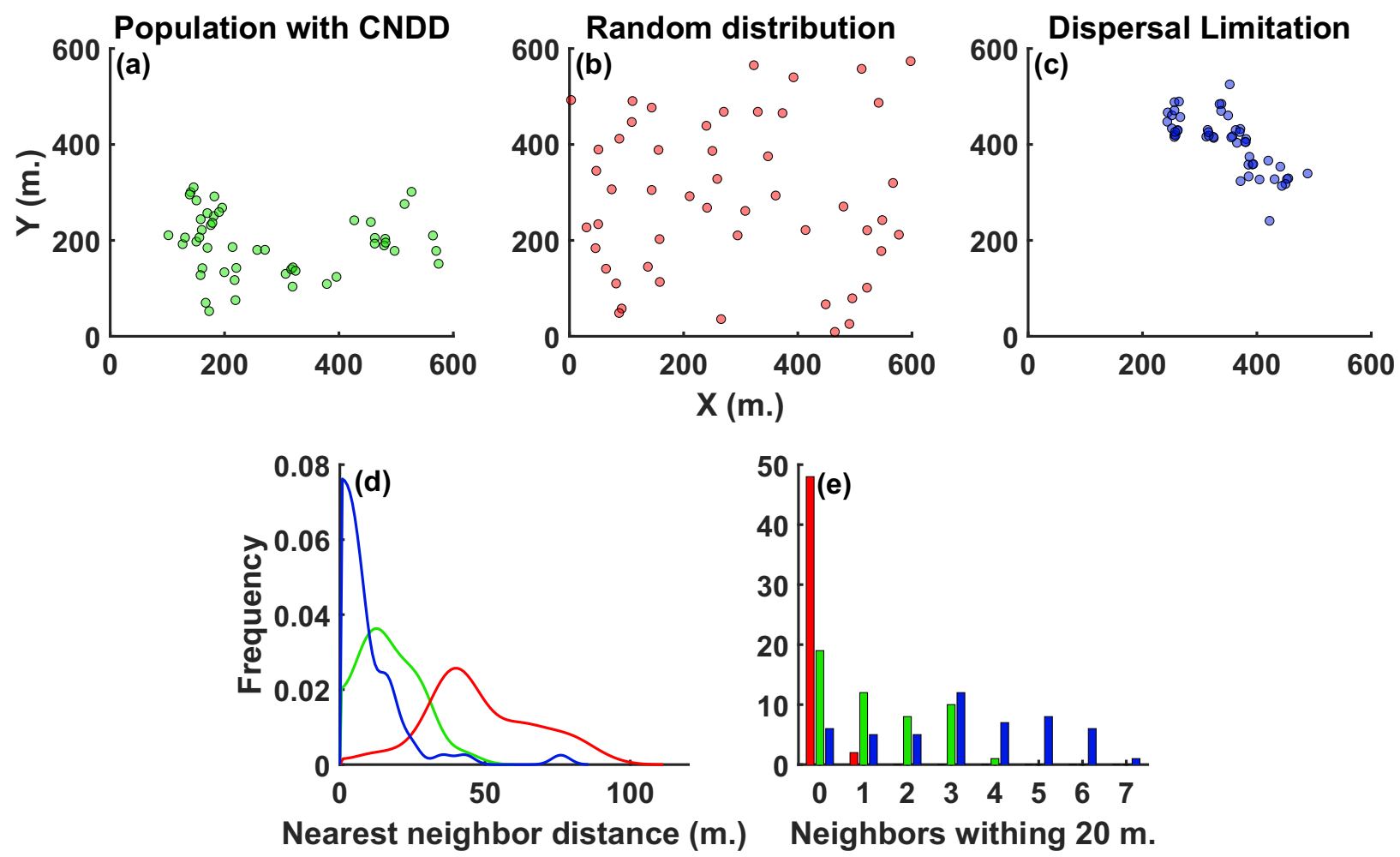

Figure 1 Comparison of the spatial point patterns of (a) a simulated population subject to both dispersal limitation and CNDD to a corresponding (b) random (CSR) and (c) Dispersal Limitation (DL) null models. The simulated population has 50 individuals, dispersal distance of 20 meters, CNDD operating to 7 meters and CNDD strength of $Q_{C}=5$ (see Simulation Models section for details). (d) presents the (kernel-smoothed) distribution of nearest neighbor distances and (e) presents the distribution of the number of neighbors within 20 meters of each individual for the three cases above. These means of the distributions in (e) and (d) are used to compute the spatial statistics (see Spatial Statistics section). In all cases, green: simulated population, red: random distribution, blue: DL. The population is more aggregated than random but less than the DL null.

Here we suggest and investigate a new approach for analyzing spatial patterns, namely comparing them with a Dispersal Limitation (DL) reference (null) model. The goal is that this reference will incorporate the aggregation generated by dispersal and hence distribute the individuals at biologically relevant scales, allowing comparisons with it to reveal the effects of biotic interactions and habitat specificity. Such comparisons are now possible due to available estimates of seed dispersal kernels (e.g., Muller-Landau et al. 2008; Wright et al. 2008).To study the characteristics of this approach and strengthen the link between spatial patterns and processes, we conduct an extensive spatially explicit simulation study of the response of measures of aggregation (with both DL and CSR as nulls) to the magnitude of CNDD and Heterospecific Negative Density Dependence (HNDD) and to the scales of CNDD and dispersal. We hypothesize that density dependence will generate overdispersion (which would increase with its magnitude) compared to DL, while a comparison to CSR will detect aggregation if dispersal is limited. We expect such overdispersion to occur on scales comparable to the scale of CNDD. We further hypothesize that patterns compared with CSR will be mostly sensitive to 
dispersal distances (Chave et al. 2002) while being insensitive to CNDD, while a comparison with DL will show substantial sensitivity to CNDD.

\section{Simulation models}

\section{The Dispersal Limitation (DL) null model}

DL is a spatially explicit, continuous space neutral simulation model aimed at answering: what are the expected species-level spatial patterns in the community if the only processes taking place are dispersal limitation and drift? Hence, it should imitate the data in terms of the landscape size, density of individuals of all species and dispersal, but include no interactions. We recommend using estimates of dispersal kernels obtained using seed trap data or mechanistic models (e.g., Muller-Landau et al. 2008; Wright et al. 2008). If species differ in their dispersal kernels or size, we recommend running a different simulation for each species with the speciesspecific dispersal kernel and density of comparable individuals.

Despite focusing the analysis on a single species, we use a multispecies zero-sum community simulation with some low level of immigration from outside. Because the species are neutral, and using a sufficient number of species, the distributions of different species could be considered as independent realizations of the null. Some limited level of immigration must therefore be included in the model to generate diversity and differences between species. Care must be taken not to choose a high immigration level that would influence spatial distributions or make common species unlikely to occur (due to the deterministic negative effect of immigration, Chisholm \& O'Dwyer 2014).

For our theoretical analysis, and to facilitate comparison, we generated the DL realizations using the same general simulation model with the same parameters as we used for communities with density dependence, but we set the magnitude of the latter to zero.

Since densities and distances between individuals strongly depend on abundance (Clark \& Evans 1954), we recommend comparing them only to samples of the DL simulation (regardless of species identity, as they are neutral) that have abundances similar to the observed. For our analyses, we binned all populations by abundance in bins of increasing width (see Appendix S4) and compared the synthetic populations to null populations of the same abundance bin.

\section{General simulation model structure}

While competition and apparent competition are often (Comita et al. 2010; Hülsmann et al. 2020) separated into CNDD and HNDD, we believe that it is more biologically justified to assume that all trees exert on all juveniles some General Negative Density Dependence (GNDD, representing competition for shared resources such as light and soil nutrients), while conspecifics also exert an added CNDD effect. Hence, in our simulations we manipulate the level of GNDD, and an added level of CNDD. The level of HNDD is equal to the level of GNDD we assume, while the level of CNDD is equal to the level of GNDD, plus the added CNDD. The situation CNDD $>$ HNDD corresponds in our formulation to added CNDD $>0$.

To generate both synthetic communities where CNDD and other forces are at work, and their null expectations, we simulate a community of sessile organisms on a 2D landscape with 
toroidal boundary conditions, inhabited by a fixed number of $J$ individuals (see Appendix S1 for a glossary). Each time step, with probability $m$, a seed arrives from the species pool to a random location, or, with probability $1-m$, a random local individual is chosen to reproduce. The offspring is dispersed using the distance distribution $f(r)$ with mean distance $D$, and upon arrival, the seed (locally produced or immigrant) establishes to become an adult with probability $\frac{1}{1+N C I}$, where $N C I$ is the Neighborhood Crowding Index.

$N C I$ is the summed contribution of the competitive effects of all individuals in the neighborhood $N$ :

$$
\begin{aligned}
& \text { (eq. 1a) } N C I=\sum_{i \in N}\left[G\left(D_{i}\right)+I_{i} C\left(D_{i}\right)\right] \text {, } \\
& \text { (eq. 1b) } G\left(D_{i}\right)=\frac{Q_{G}}{1+\left(\frac{D_{i}}{\Delta_{G}}\right)^{\alpha_{G}}}, \\
& \text { (eq. 1c) } C\left(D_{i}\right)=\frac{Q_{C}}{1+\left(\frac{D_{i}}{\Delta_{C}}\right)^{\alpha_{C}}},
\end{aligned}
$$

where an individual $i$ at distance $D_{i}$ exerts $G N D D, G\left(D_{i}\right)$, and added CNDD, $C\left(D_{i}\right)$ if it is a conspecific, with $I_{i}$ being an indicator variable for the latter. $Q_{G}$ and $Q_{C}$ are the per-capita magnitudes of GNDD and added CNDD, respectively. $\Delta_{G}$ and $\Delta_{C}$ are the distances at which GNDD and added CNDD, respectively, fall to half of their magnitude, and $\alpha_{G}$ and $\alpha_{C}$ set how rapidly this decay occurs, with higher values meaning the competition force remains high out to distances closer to to $\Delta_{G}$ and $\Delta_{C}$, and then declines more quickly with distance from there (see Figure S1 for a visualization of eq. 1b-1c). We always consider the neighborhood $N$ to consist of all individuals up to a distance of $2 \cdot \Delta_{C}$ or 15 meters, whichever is greater, from the seed. Following successful recruitment, a random individual is chosen to die.

\section{Simulated parameters}

\section{General settings and dispersal}

To roughly resemble a tropical forest plot (where such patterns have been extensively studied), we set the landscape to be a quadrat with edge length $L=600$ meters and $J=5,500$ individuals, resembling the density of adult trees in Barro Colorado Island, Panama (Condit et al. 2019). Immigration is set to occur with probability $m=5 \cdot 10^{-4}$ from a regional pool of $S=300$ species with equal frequency. $m$ was chosen to be low to avoid an effect on local spatial patterns, but still high enough to maintain many species with variable abundances.

In line with several empirical works on dispersal, we set $f(r)$ to be a fat-tailed 2DT with 3 degrees of freedom (Clark et al. 1999; Muller-Landau et al. 2008). We study a wide regime of parameters to test the robustness of the observed patterns and to examine the sensitivity of different statistics to changes in the parameters. 


\section{GNDD and CNDD}

We set the distance of GNDD to resemble the overall density in tropical forests: $\Delta_{G}=4.5$ meters, representing roughly the crown diameter of adult trees, and $\alpha_{G}=5$, so that at a distance of 20 meters the effect drops to almost zero $\left(\sim 0.0005 \mathrm{Q}_{\mathrm{G}}\right.$, Uriarte et al. 2005).

Four mean dispersal distances $(D)$ and CNDD distances $\left(\Delta_{C}\right)$ are used: 7 meters, 20 meters, 60 meters and 3000 meters (effectively infinite distance). For CNDD, we simulated each of these four $\Delta_{C} \mathrm{~S}$ with a corresponding steepness parameter value $\left(\alpha_{C}\right): 5,6,7,10$, respectively (each chosen to create a relatively quick decline in CNDD at $\Delta_{C}$, which requires a higher value for higher $\Delta_{C}$ ). We assume that CNDD is operating to a larger distance than GNDD because natural enemies, the primary hypothesized cause of CNDD (Hülsmann et al. 2020), are capable of dispersal and movement. The dispersal distances are also set to represent realistic variation in forests (Clark et al. 1999; Muller-Landau et al. 2008).

To study the effect of the magnitude of CNDD and GNDD, we use all combinations of $Q_{G}$ and $Q_{C}$ of $0,0.2,1$ and 5 .

To consider scenarios where different species suffer different levels of CNDD (as found by e.g., Comita et al. 2010; Lebrija-Trejos et al. 2016), we further run simulations where each species has its own value of $Q_{C}, Q_{C}^{\prime}$, and these values are distributed between $Q_{C \min }$ and $Q_{C \min }$ $+Q_{\text {Crange }}$ equidistantly. For these simulations, we used the same dispersal and CNDD distances as above, excluding the infinite distance cases. We used $Q_{C \min }=1$ and two levels of $Q_{\text {Crange }}, 1$ and 5. $Q_{G}$ was assumed to be 0.2 in these simulations.

\section{Simulation time and sampling}

Each simulation was initiated as a random placement of $J$ individuals sampled from the pool and was given 3000 generations ( $J$ time steps each) to equilibrate. For the simulations with fixed CNDD, four levels of the four parameters $\left(D, \Delta_{C}, Q_{G}\right.$ and $\left.Q_{C}\right)$ were run. Following that, 3000 snapshots of each simulation were taken, with 10 generations between snapshots. For the simulations with variable CNDD, three levels of $D$ and $\Delta_{C}$ and two levels of $Q_{\text {Crange }}$ were used. 9 independent runs were used for each regime, each with 1000 snapshots taken after equilibration and 50 generations between snapshots,

In the results we present the mean values of the statistics for all populations with abundance $\geq 5$ recorded in a parameter regime, sometimes subdivided by abundance when this is indicated.

\section{Spatial statistics}

The two most common approaches to summarizing univariate point patterns are to examine the distances of individuals to their nearest neighbors (Figure 1d) or the density of neighbors around each individual (Figure 1e) at different distances, which are classically compared to their values under CSR. In particular, the Clark-Evans $(C E)$ statistic compares the average distance of 
an individual to its conspecific nearest neighbor in the observed data, $N N D_{o b s}$, to its expectation under CSR, which equals $\frac{1}{2 \sqrt{\lambda}}$, where $\lambda$ is the density of the species (Clark \& Evans 1954):

(eq. 2) $C E=2 N N D_{o b s} \sqrt{\lambda}$.

If $C E>1$ the population is overdispersed compared to CSR, while $C E<1$ indicates aggregation.

We propose the Excess neighbor Distance $(E D)$ statistic, an analogous measure that is symmetric on a logarithmic scale. Distances are compared to the mean nearest neighbor distance in realizations of the DL model that have similar abundance, $N N D_{\text {null }}$ :

(eq. 3) $E D=\log \left(N N D_{\text {obs }}\right)-\overline{\log \left(N N D_{\text {null }}\right)}$.

The averaging is done over different realizations of DL. Positive values of $E D$ indicate overdispersion with respect to DL while negative values indicate aggregation.

To study the density of neighbors, many works use the Relative Neighborhood Density statistic (RND, also known as pair-correlation function; Condit et al. 2000; Wiegand \& Moloney 2013). $R N D(r)$ compares the observed number of conspecific neighbors that an average individual has between distances $r$ and $r+\Delta r, N_{o b s}(r)$, to its expectation under CSR, $A(r) \lambda$, where $A(r)$ is the area of the annulus with inner and outer radii $r$ and $\mathrm{r}+\Delta r$, respectively:

(eq. 4) $R N D(r)=\frac{N_{o b s}(r)}{A(r) \lambda}$.

If $R N D(r)>1$, the population is aggregated compared to CSR at distances $\mathrm{r}$ to $\mathrm{r}+\Delta r$, while $R N D(r)<1$ indicates overdispersion. We propose the analogous Excess neighborhood Abundance $(E A)$ statistic,

(eq. 5) $\quad E A(r)=\log \left(N_{\text {obs }}(r)\right)-\overline{\log \left(N_{\text {null }}(r)\right)}$,

where $N_{\text {null }}(r)$ is the analog of $N_{\text {obs }}(r)$ in a comparable realization (in terms of abundance) of the DL null. Positive values of $E A$ indicate aggregation with respect to the DL, while negative values indicate overdispersion. In practice, we study $E A$ and $R N D$ at 10-meter distance bins, and we also focus on a circle of 20 meters $(E A(20)$ and $R N D(20))$.

While direct analogs of RND and the Clark-Evans statistics could be devised for use with DL, $E D$ and $E A$ are constructed on a logarithmic scale, to create symmetry between aggregation and overdispersion to better capture the variation in spatial dispersion (Wiegand et al. 2007). Intuitively, if $E D$ or $E A(r)$ have a value of $x$, it indicates that the average nearest neighbor distance or number of conspecific neighbors at distance $r$, respectively, are larger by $\mathrm{e}^{x}$ then their expectation, which is the geometric mean over realizations of the null. The statistical significance of both $E A$ and $E D$ can be evaluated by comparing $\log \left(N_{o b s}(r)\right)$ or $\log \left(N N D_{o b s}\right)$ with the distribution of their analogs under the nulls with a two-sided test. 


\section{Results and discussion}
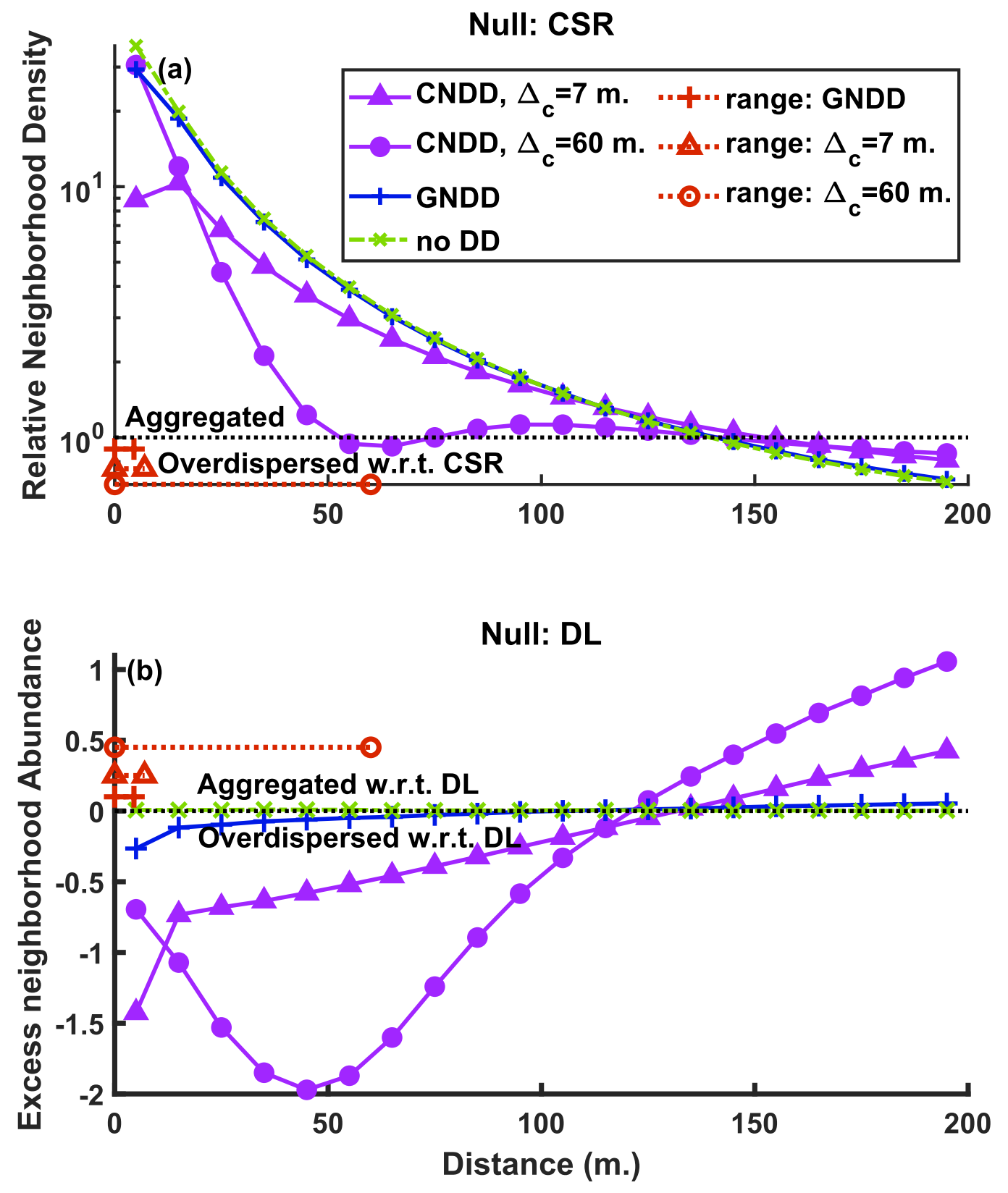

Figure 2Aggregation and overdispersion of simulated populations at different scales, compared with (a) the Complete Spatial Randomness (CSR) null, using Relative Neighborhood Abundance (Eq. 4), and (b) the Dispersal Limitation (DL) null, using Excess Abundance (Eq. 5). Mostly aggregation is seen in (a), where in (b) the overdispersion created by CNDD is made apparent, and it is seen out to large scales. Dispersal distance $(D)$ in all cases is 20 meters. Purple curves with triangle and circle symbols are cases with CNDD only $\left(Q_{G}=0, Q_{C}=5\right)$ operating to distances $\left(\Delta_{C}\right)$ of 7 and 60 meters, respectively. The blue and green curves represent simulations with only $\operatorname{GNDD}\left(Q_{G}=5, Q_{C}=0\right)$ and no density dependence $\left(Q_{G}=0, Q_{C}=0\right)$, respectively. Full correspondence with the null is represented with a grey dotted line. Red lines present the distance scale of density dependence of the corresponding CNDD or GNDD scenario (indicated by the symbol). 


\section{Overdispersion and its scale}

As we expected, dispersion patterns (averaged across species) appear aggregated compared to CSR as long as dispersal is not global. (Figures 2a, S3, S5). Since typical clumps are large, this aggregation extends up to scales $\sim 150$ meters (Figure $2 \mathrm{a}$ ). On the other hand, when compared to DL, Both GNDD and added CNDD, separately and in combination, generate overdispersion relative to DL (Figures 2-4, S2, S4). However, counter to our expectations, this overdispersion occurs at scales much larger than the scales of density dependence or dispersal (Figure $2 \mathrm{~b}$ ), and even GNDD by itself (acting at a distance $\sim 5$ meters) can create overdispersion on scales of dozens of meters.

We believe this result represents the emergence of large-scale structure from local interactions, which is common in many ecosystems (Levin 1992). The neighbors of each individual tend to be overdispersed, but so are their neighbors, and so forth. Hence, if large-scale overdispersion is observed in nature, it likely stems from CNDD acting on considerably shorter scales.

Another interesting finding is the appearance of a trough in both the $E A(r)$ and $R N D(r)$ curves when added CNDD operates at long distances (dozens of meters), roughly at the typical scale of CNDD. This is the result of the emergence of clumps of a few individuals that are surrounded by large vacant areas (Figure S6).

Note that the high $E A$ and low $R N D$ at large distances are an unavoidable result: since the overall number of individuals is fixed, having more/less neighbors than expected at short distances necessitates having less/more at longer distances. Still, these patterns are typically interpreted focusing on short distances (Wiegand \& Moloney 2013).

See appendix S2 for an analysis of the effects of added CNDD distance scale and dispersal distance scale on spatial statistics.

\section{Effects of the magnitude of CNDD and GNDD}

As we expected, an increase in both GNDD and added CNDD, when they operate separately, increases overdispersion compared to DL (Figures 3, S7a,b). However, the effect of GNDD by itself on overdispersion in the absence of added CNDD is much weaker than the effect of added CNDD by itself (Compare the effect of changes in $Q_{G}$ when $Q_{C}=0$ to changes in $Q_{C}$ when $Q_{G}=0$ in Figure 3a,b). Moreover, when both GNDD and added CNDD operate (as is expected in natural systems), their effects counteract each other: if added CNDD is strong, the effect of increasing $\mathrm{Q}_{\mathrm{G}}$ is reversed to reducing overdispersion. If GNDD is strong, increasing $\mathrm{Q}_{\mathrm{C}}$ still increases overdispersion, but at a slower rate (Figures 3a,b, S7a,b). Overall, the strongest overdispersion can only be observed when added CNDD >> GNDD and therefore strong overdispersion could indicate this scenario (bright yellow colors indicating high overdispersion occur only in the lower right in Figures 3a,b). Finally, using CSR as null, $C E$ shows a unimodal shaped dependence or limited dependence on $Q_{C}$ (Figure $3 \mathrm{~d}$ ), while the behavior of $R N D(20)$ is consistent with $E D$ and $E A(20)$ (Figure $\mathrm{S} 7 \mathrm{~d}$ ). 


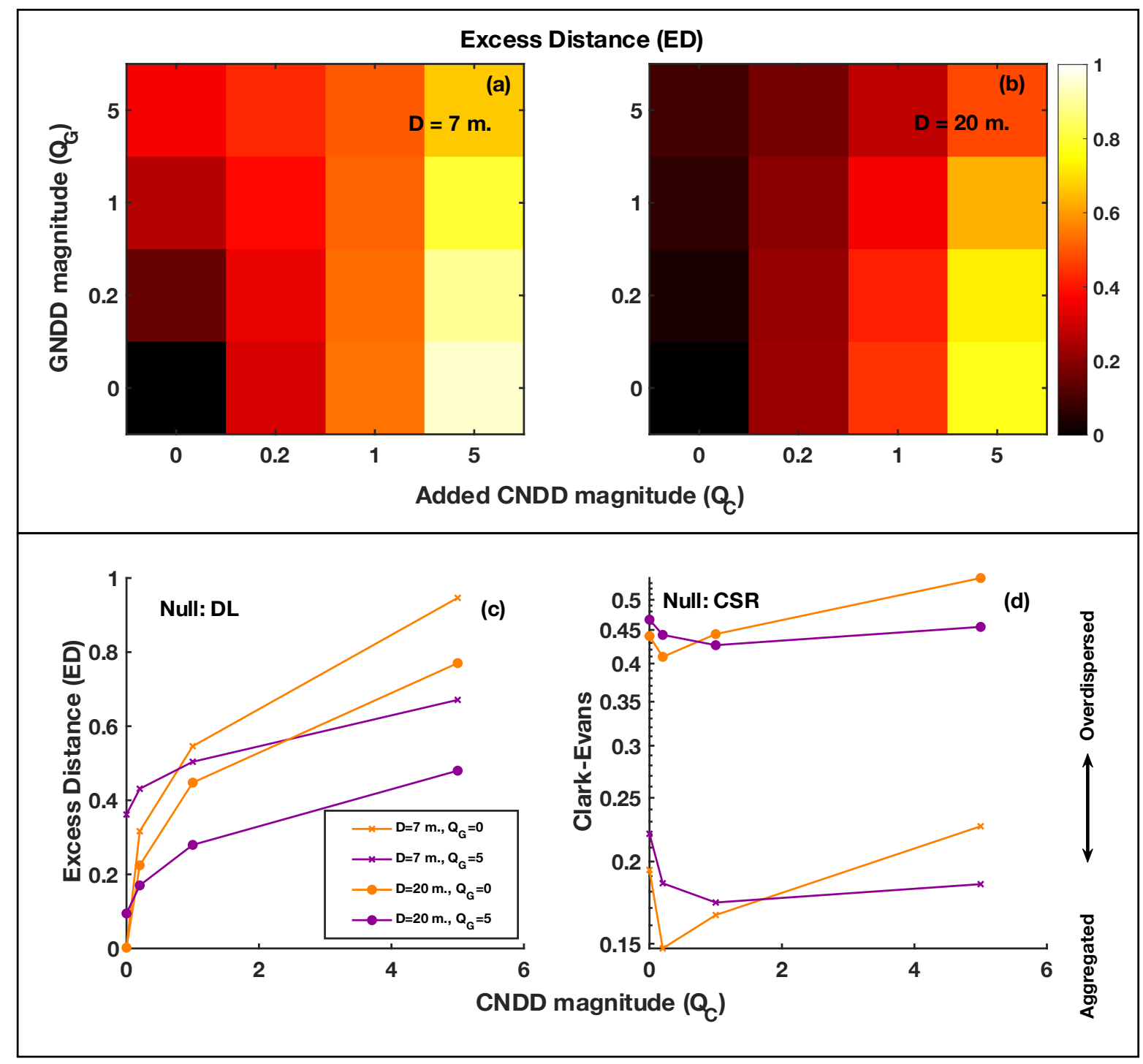

Figure 3 Effect of the magnitude of $\operatorname{GNDD}\left(Q_{G}\right)$ and added $\operatorname{CNDD}\left(Q_{C}\right)$ on nearest neighbor statistics. In all cases $\Delta_{C}=20$ meters. The colors of (a) and (b) represents the Excess nearest neighbor Distance ( $E D$, eq. 3$)$ in response to a combination of GNDD and CNDD for dispersal distances $(D)$ of 7 meters and 20 meters, respectively. Furthermore, the effect of $Q_{C}$ on (c) $E D$ and on (d) the Clark-Evans statistic (eq. 2), with the latter using CSR as null, are presented when $Q_{G}=0$ (orange) or $Q_{G}=5$ (purple). GNDD alone can produce much lower overdispersion than added CNDD. GNDD counteracts the effect of added CNDD and the strongest overdispersion is observed when CNDD is maximal and GNDD is minimal.

These results can be understood by considering the spatial uniformity of seed mortality. GNDD is caused by all individuals of all species similarly, causing a relatively spatially uniform mortality across the plot, while added CNDD generates "repulsion" only from conspecifics. Consequently, GNDD cannot cause high species-specific overdispersion, and it can homogenize the non-uniform mortality caused by CNDD, reducing the latter's effect. Analogously, if CNDD is not distance dependent $\left(\Delta_{C}=\infty\right)$, it will homogenize the local effect of GNDD, and in this 
situation increasing $Q_{C}$ may reduce overdispersion (Figure S8). Finally, the "peculiar" behavior of the Clark-Evans statistic, whereas increasing $Q_{C}$ may increase aggregation, is probably due to the high sensitivity of this statistic to abundance.

\begin{tabular}{|c|c|c|}
\hline$\times$ & $\begin{array}{l}\mathrm{D}=7 \mathrm{~m} ., \Delta_{C}=7 \mathrm{~m} . \\
\mathrm{D}=7 \mathrm{~m} ., \Delta_{C}=60 \mathrm{~m} \\
\mathrm{D}=20 \mathrm{~m} ., \Delta_{C}=20 \mathrm{~m}\end{array}$ & $\begin{array}{l}\times D=60 \mathrm{~m} ., \Delta_{c}=7 \mathrm{~m} . \\
. \quad D=60 \mathrm{~m} ., \Delta_{c}=60 \mathrm{~m} .\end{array}$ \\
\hline
\end{tabular}
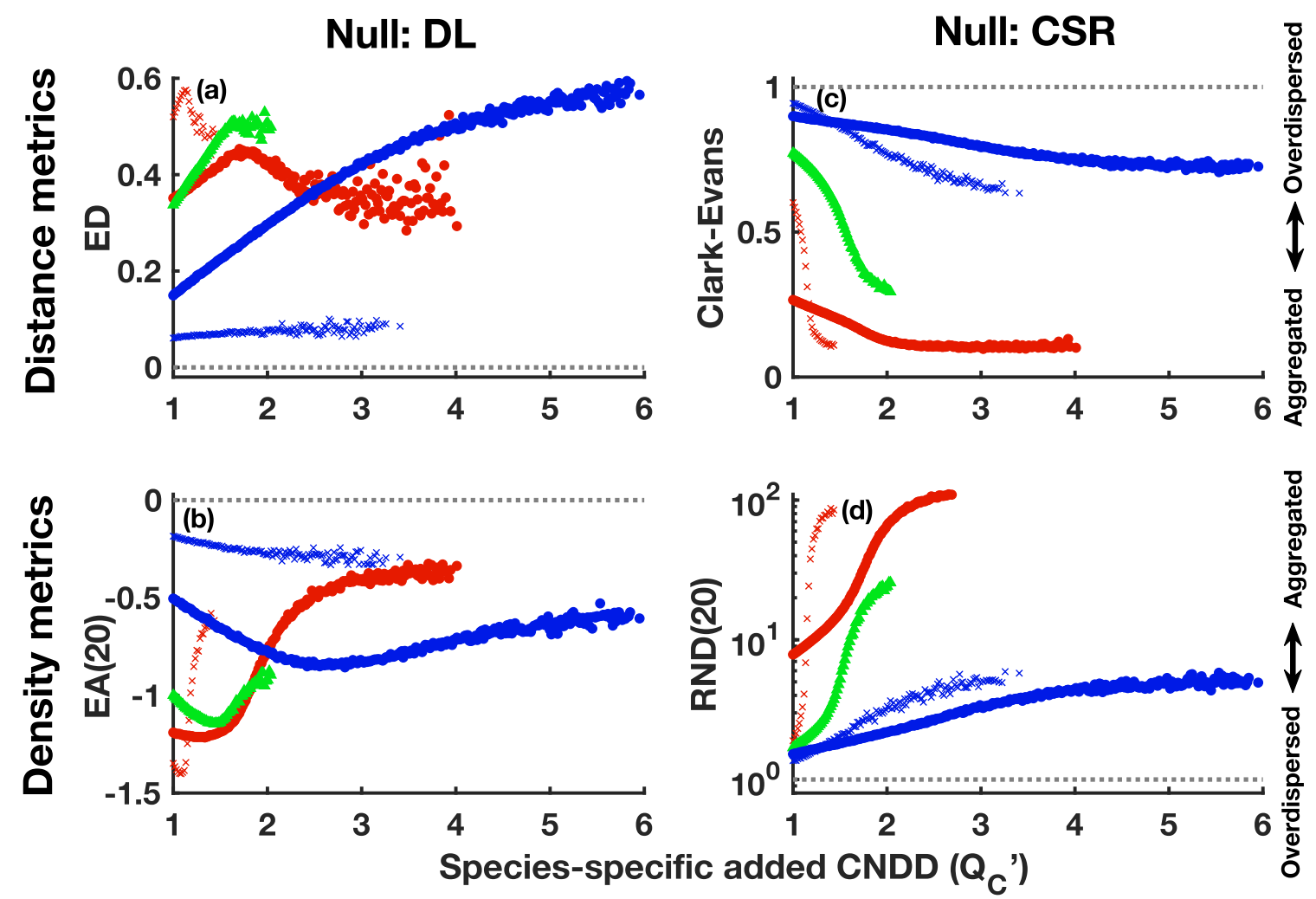

Figure 4 Effect of the species-specific magnitude of CNDD $\left(Q_{C^{\prime}}\right)$ on spatial statistics: (a) Excess nearest neighbor Distance $(E D)$; (b) Excess neighborhood Abundance within 20 meters $(E A(20))$; (c) the Clark-Evans nearest neighbor statistic and (d) Relative Neighborhood Density within 20 meters $(R N D(20))$. The top row examines nearest neighbor distance statistics while the bottom row shows neighborhood density. The statistics on the left column have DL as reference while the right column has CSR. Different colors and symbols represent regimes with different levels of dispersal distance $(D)$ and CNDD distance $\left(\Delta_{C}\right)$, respectively. In all cases, GNDD magnitude $\left(Q_{G}\right)$ is 0.2 and CNDD has a minimum value $Q_{C \min }=1$ and $Q_{\text {Crange }}=5$. Species with fewer than 500 observations were discarded, hence, some parameter regimes do not have observations for high $Q_{C}$ '. Overdispersion depends unimodally on $Q_{C}$ ' when using the DL null, while compared to CSR species with higher $Q_{C}$ ' appear more aggregated.

\section{Effects of variation in abundance and CNDD across species}

Thus far we analyzed the spatial statistics averaged across species. How do these patterns depend on species' abundance? From the perspective of CSR, there is a strong effect of reduced 
aggregation with abundance, as is commonly found in tropical forests (Appendix S3, Condit et al. 2000). From the perspective of DL, the relationship is more complex and often unimodal or flat. See appendix S3 for more details and for an analysis of the underlying mechanisms.

Next, we examined the case when species differ in their species-specific susceptibility to CNDD $\left(Q_{C}{ }^{\prime}\right)$. We hypothesized that species that have higher $Q_{C}$ ' would be more overdispersed. However, our simulations show that, from the perspective of statistics using DL as a null, the effect of $Q_{C}$ ' is unimodal with species of intermediate $Q_{C}$ ' being the most overdispersed, but sometimes only the increasing or decreasing phase is observed (Figure 4a,b). Qualitatively similar results are observed for lower values of $Q_{\text {Crange }}$ (Figure S9). Surprisingly, statistics that use CSR as a null suggest that species with higher $Q_{C}$ ' are more aggregated (Figure $4 \mathrm{c}, \mathrm{d}$ ).

These non-trivial relationships are a result of the effect of $Q_{C}$ ' on abundance: species with higher $Q_{C}$ ' are substantially rarer in our model. Consequently, increasing $Q_{C}$ ' affects dispersion both directly, increases the repulsion effect (as in Figure 3), and indirectly, by making species rarer. With the statistics that use CSR as reference, the reduced abundance causes a sharp increase in aggregation, creating the counter-intuitive increase in aggregation with CNDD. With the statistics that are compared to DL, the typical effect of abundance is unimodal (see Appendix $\mathrm{S} 3$ ), and this adds to the increase in repulsion with stronger $Q_{C}$ ' to shape the unimodal effect of $Q_{C}$ ' on overdispersion.

\section{Sensitivity to different parameters}

Figure 5 presents the effect of changing one parameter at a time from an intermediate level to a high level, while keeping the others fixed, across the distributions of the other parameters. First, the statistics that use CSR as null are primarily sensitive to dispersal distance, far beyond their sensitivities to competition (in line with the findings of Chave et al. 2002). When using DL as null, the $E D$ statistic is sensitive to the different parameters to a comparable degree, while $E A(20)$ is primarily sensitive to the magnitude of CNDD, more than to other parameters. Finally, all statistics are weakly affected by GNDD. Hence, we expect the DL null to have high capacity to detect CNDD in empirical data, especially with the EA(20) statistic, unlike the CSR null.

\section{Conclusions and overview}

Ecologists have long sought an approach to gaining insight into process from spatial patterns within species, but this goal has been elusive. Our theoretical analysis demonstrates the usefulness of spatial patterns for understanding the cumulative effect of density dependent interactions on adult individuals in natural communities, once they are compared with predictions based on dispersal limitation, rather than complete spatial randomness. First, it is of fundamental importance to evaluate the magnitude of added CNDD, or, equivalently, the degree to which CNDD > HNDD, as this is the requirement for stabilizing niche differences, which may lead to coexistence if fitness differences are sufficiently small (Hülsmann et al. 2020). Our analysis reveals that high levels of overdispersion compared to DL are only possible if added CNDD is substantial and can be used to detect this ecologically important situation. This stems 
from the combination of two factors: first, in the absence of added CNDD (when CNDD = HNDD $=$ GNDD, left column of Figures 3a,b), GNDD is only capable of generating limited values of overdispersion, at least for dispersal distances of 20 meters (typical of tropical forests, Muller-Landau et al. 2008). On the other hand, much higher levels of overdispersion are enabled by added CNDD. Second, GNDD counteracts added CNDD, so if both are present, overdispersion will be reduced compared to a situation when GNDD is weak. Hence, strong GNDD and weak added CNDD are inconsistent with high overdispersion.

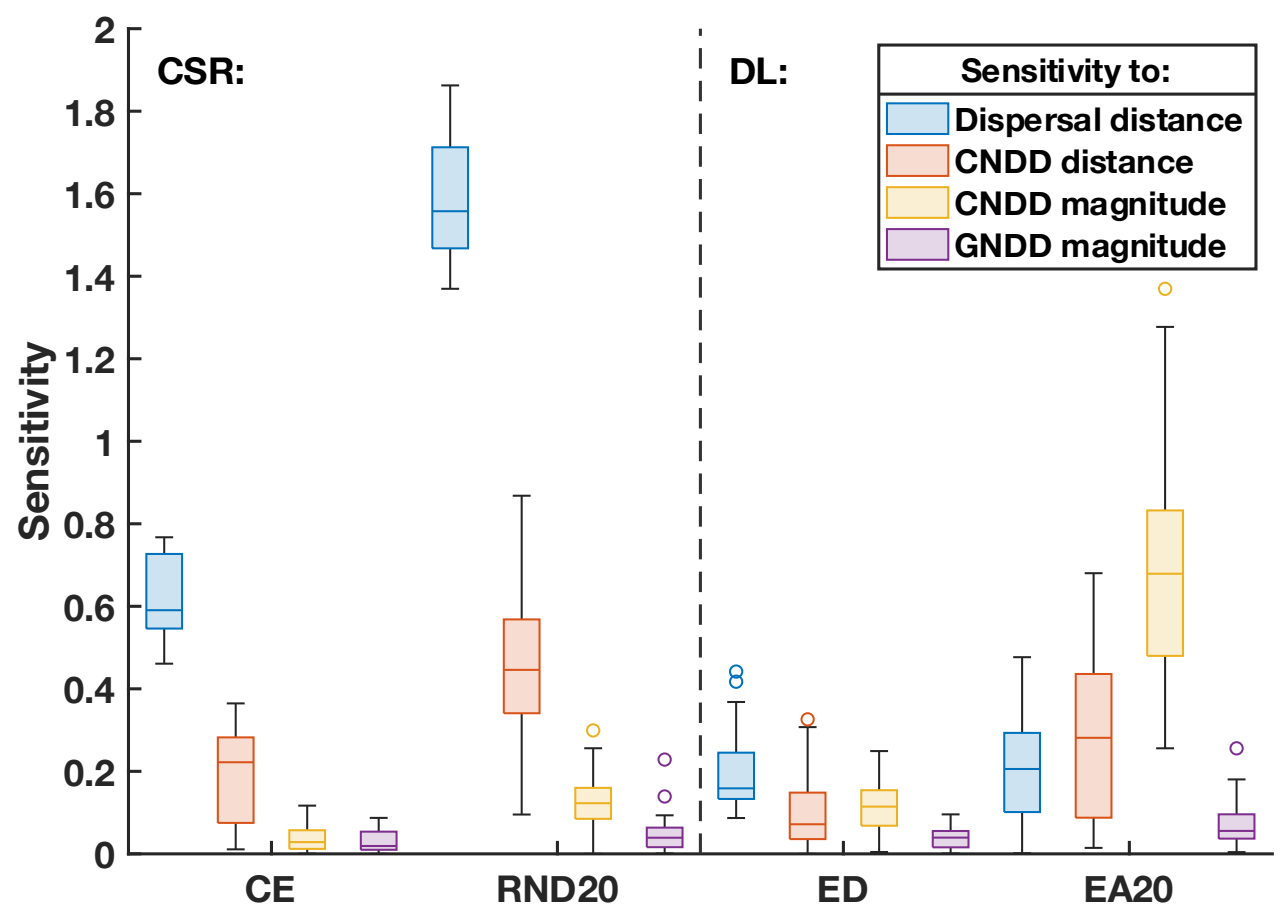

Figure 5 Sensitivity of the statistics using DL and CSR as reference to changes in the simulation parameters. Sensitivities are calculated as the effect on the mean value of the statistic (on a logarithmic scale, hence $R N D(20)$ and Clark-Evans are log transformed for comparison) of changing one parameter at a time from an intermediate level $\left(\mathrm{D}=20 \mathrm{~m}\right.$. or $\Delta_{C}=20 \mathrm{~m}$. or $Q_{C}=0.2$ or $\left.Q_{C}=0.2\right)$ to a higher level $\left(\mathrm{D}=60 \mathrm{~m} ., \Delta_{C}=60 \mathrm{~m}\right.$., $Q_{C}=$ $1, Q_{C_{-}}=1$, respectively). The distributions of these sensitivities, each calculated by comparing a pair of simulations differing in the parameter of interest, is presented across all the values of the other parameters. Statistics based on CSR have low sensitivity to CNDD and high sensitivity to dispersal distance, while statistics based on DL are much more sensitive to CNDD.

Among the information that can be obtained from spatial patterns are the relative scales of CNDD and dispersal. If dispersal acts on longer distances than CNDD, as is believed (Janzen 1970), we show that $E A(r)$ should decrease with $r$ monotonically. Moreover, it is possible to correlate measures of CNDD magnitude (e.g., Comita et al. 2010; Lebrija-Trejos et al. 2016) with values of $E D$ and $E A$ to test whether specific measures of CNDD are associated with adult distributions. 
Another factor that can have a substantial effect on species' distributions is habitat specificity. We did not include this important factor in our analyses since there are multiple established methods to quantify habitat specificity using spatial distributions (e.g., Harms et al. 2001; Wiegand et al. 2007; Flugge et al. 2014). We hypothesize that if the habitat is patchy, seeds would be lost to unsuitable habitats and this will reduce overdispersion and potentially even create aggregation compared to DL. Such aggregation may further be reduced at short distances owing to CNDD. We view such aggregated patterns as much more informative than aggregation compared to CSR. Overall, the analysis of spatial patterns of adults can complement analyses of demography for understanding community dynamics. Specifically, explaining the variation in $E D$ and $E A(20)$ among species can detect the relative importance of factors shaping species' distributions, and our work suggests that such analyses should include abundance and dispersal distance as covariates, since they have a substantial effect on these statistics.

Nevertheless, our results emphasize that spatial patterns observed in nature are a complex emergent phenomenon. While we initially expected that the magnitude and scale of overdispersion could serve as a measure of the magnitude and scale of CNDD, our results show this is not straightforward: even with all else being equal, we find that the relationship between CNDD and overdispersion may not be monotonic, so that species subject to stronger CNDD may be less overdispersed (Figure 4), let alone if they differ by other factors such as dispersal distance. The scale of emerging population overdispersion is also found to exceed the scale of CNDD caused by individuals. Finally, overdispersion compared to DL is the combined product of GNDD and added CNDD that counteract each other, up to the point that each could reduce overdispersion under specific conditions.

A limitation of our approach is its dependence on reliable estimates of dispersal distance that are difficult to obtain. We recommend that empirical analyses comparing spatial patterns to DL should always test the robustness of results by using multiple dispersal kernels, incorporating this uncertainty. Assuming too long dispersal distance is expected to bias estimates of overdispersion downwards, which could be more conservative if the goal is to detect CNDD. Finally, it is possible to analyze what dispersal distance is required to obtain the observed patterns, and if that distance is unrealistically large (e.g., 200 meters for trees) this could serve as evidence for CNDD.

The use of CSR as a reference for dispersion patterns is the simplest analysis of spatial patterns, that has been done for over a hundred years (Gleason 1920), often with the aim of detecting the signals of habitat specificity and biotic interactions (Condit et al. 2000; Plotkin et al. 2000; Bagchi et al. 2011). However, previous analyses (Chave et al. 2002) as well as this work, show that dispersal limitation will almost inevitably obscure the signal of these factors when using CSR. Moreover, we show that the expected dependence of spatial patterns compared to CSR on species' properties such as the magnitude of CNDD makes little sense in some situations, likely due to their sensitivity to abundance. All this is due to considering the reference at a biologically irrelevant scale - the scale of the survey plot, over which the individuals are randomly distributed with no biological justification. Conversely, with our approach, 
distributions are compared to their expectations if only dispersal and drift were operating. Hence, we believe that DL sets the biologically relevant and interpretable reference for spatial patterns rooted in ecological theory, offering a complimentary definition of "aggregation" and “overdispersion". we hope this work will facilitate its application to natural populations.

\section{Acknowledgements}

We thank S.J. Wright, Ronen Kadmon and members of the Ostling lab for helpful comments on the manuscript. This research was supported in part through computational resources and services provided by Advanced Research Computing at the University of Michigan, Ann Arbor. MK was supported by a Michigan Life Sciences Fellowship, the Zuckerman STEM Leadership Program, funds from MCubed and the Associate Professor Support Fund at the University of Michigan. JKL acknowledges Adrian College and the University of Michigan for sabbatical support.

\section{References}

1.

Bagchi, R., Henrys, P.A., Brown, P.E., Burslem, D., Diggle, P.J., Gunatilleke, C.V.S. et al. (2011). Spatial patterns reveal negative density dependence and habitat associations in tropical trees. Ecology, 92, 1723-1729.

2 .

Begon, M. \& Townsend, C.R. (2020). Ecology: from individuals to ecosystems. John Wiley \& Sons.

3.

Brown, C., Illian, J.B. \& Burslem, D. (2016). Success of spatial statistics in determining underlying process in simulated plant communities. Journal of Ecology, 104, 160-172.

4.

Cadotte, M.W. \& Tucker, C.M. (2017). Should Environmental Filtering be Abandoned? Trends in Ecology \& Evolution, 32, 429-437.

5.

Chave, J., Muller-Landau, H.C. \& Levin, S.A. (2002). Comparing classical community models:

6. Theoretical consequences for patterns of diversity. American Naturalist, 159, 1-23.

Chisholm, R.A. \& O'Dwyer, J.P. (2014). Species ages in neutral biodiversity models. Theoretical Population Biology, 93, 85-94.

7.

Clark, J.S., Silman, M., Kern, R., Macklin, E. \& HilleRisLambers, J. (1999). Seed dispersal near and far: Patterns across temperate and tropical forests. Ecology, 80, 1475-1494.

8.

Clark, P.J. \& Evans, F.C. (1954). Distance to nearest neighbor as a measure of spatial relationships in populations. Ecology, 35, 445-453.

9.

Comita, L.S., Muller-Landau, H.C., Aguilar, S. \& Hubbell, S.P. (2010). Asymmetric Density Dependence Shapes Species Abundances in a Tropical Tree Community. Science, 329, 330-332. 
10 .

Condit, R., Ashton, P.S., Baker, P., Bunyavejchewin, S., Gunatilleke, S., Gunatilleke, N. et al. (2000). Spatial patterns in the distribution of tropical tree species. Science, 288, 14141418.

11 .

Condit, R., Pérez, R., Aguilar, S., Lao, S., Foster, R. \& Hubbell, S. (2019). Complete data from the Barro Colorado 50-ha plot: 423617 trees, 35 years. URL https://doi.

12. org/10.15146/5xcp-0d46.

Detto, M., Visser, M.D., Wright, S.J. \& Pacala, S.W. (2019). Bias in the detection of negative

13. density dependence in plant communities. Ecology Letters, 22, 1923-1939.

Flugge, A.J., Olhede, S.C. \& Murrell, D.J. (2014). A method to detect subcommunities from multivariate spatial associations. Methods in Ecology and Evolution, 5, 1214-1224.

14.

Gleason, H.A. (1920). Some applications of the quadrat method. Bulletin of the Torrey Botanical Club, 47, 21-33.

15.

Gotelli, N.J. \& Ulrich, W. (2012). Statistical challenges in null model analysis. Oikos, 121, 171180.

16.

Harms, K.E., Condit, R., Hubbell, S.P. \& Foster, R.B. (2001). Habitat associations of trees and shrubs in a 50-ha neotropical forest plot. Journal of Ecology, 89, 947-959.

17.

Hubbell, S.P. (1979). Tree dispersion, abundance, and diversity in a tropical dry forest. Science, 203, 1299-1309.

18.

Hubbell, S.P. (2001). The unified neutral theory of biodiversity and biogeography. Princeton University Press.

19.

Hubbell, S.P., Foster, R.B., O'Brien, S.T., Harms, K.E., Condit, R., Wechsler, B. et al. (1999). Light-gap disturbances, recruitment limitation, and tree diversity in a neotropical forest. Science, 283, 554-557.

20.

Hülsmann, L., Chisholm, R.A. \& Hartig, F. (2020). Is Variation in Conspecific Negative Density Dependence Driving Tree Diversity Patterns at Large Scales? Trends in Ecology \& Evolution.

21.

Janzen, D.H. (1970). Herbivores and the number of tree species in tropical forests. American Naturalist, 104, 501-528.

22.

Johnson, D.J., Beaulieu, W.T., Bever, J.D. \& Clay, K. (2012). Conspecific Negative Density Dependence and Forest Diversity. Science, 336, 904-907.

23. 
Kalyuzhny, M., Flather, C.H., Shnerb, N.M. \& Kadmon, R. (2022). A framework for quantifying deviations from dynamic equilibrium theory. Global Ecology and Biogeography, 31, 183-195.

24.

Kraft, N.J.B., Adler, P.B., Godoy, O., James, E.C., Fuller, S. \& Levine, J.M. (2015). Community assembly, coexistence and the environmental filtering metaphor. Functional Ecology, 29, 592-599.

25 .

Law, R., Murrell, D.J. \& Dieckmann, U. (2003). Population growth in space and time: Spatial logistic equations. Ecology, 84, 252-262.

26.

Lebrija-Trejos, E., Reich, P.B., Hernandez, A. \& Wright, S.J. (2016). Species with greater seed mass are more tolerant of conspecific neighbours: a key driver of early survival and 27. future abundances in a tropical forest. Ecology Letters, 19, 1071-1080.

Levin, S.A. (1992). The Problem of Pattern and Scale in Ecology. Ecology, 73, 1943-1967. 28.

Losos, J.B. \& Ricklefs, R.E. (2010). The theory of island biogeography revisited. Princeton University Press.

29.

Mangan, S.A., Schnitzer, S.A., Herre, E.A., Mack, K.M.L., Valencia, M.C., Sanchez, E.I. et al. (2010). Negative plant-soil feedback predicts tree-species relative abundance in a tropical forest. Nature, 466, 752-U710.

30.

McIntire, E.J.B. \& Fajardo, A. (2009). Beyond description: the active and effective way to infer processes from spatial patterns. Ecology, 90, 46-56.

31.

Mittelbach, G.G. \& McGill, B.J. (2019). Community ecology. Oxford University Press.

32.

Molofsky, J., Bever, J.D. \& Antonovics, J. (2001). Coexistence under positive frequency dependence. Proceedings of the Royal Society B-Biological Sciences, 268, 273-277.

33.

Muller-Landau, H.C., Wright, S.J., Calderon, O., Condit, R. \& Hubbell, S.P. (2008). Interspecific variation in primary seed dispersal in a tropical forest. Journal of Ecology,

34. 96, 653-667.

Pielou, E.C. (1962). The Use Of Plant-to-neighbour Distances For The Detection Of Competition. Journal of Ecology, 50, 357-367.

35.

Plotkin, J.B., Potts, M.D., Leslie, N., Manokaran, N., LaFrankie, J. \& Ashton, P.S. (2000). Species-area curves, spatial aggregation, and habitat specialization in tropical forests. Journal of Theoretical Biology, 207, 81-99.

36.

Seidler, T.G. \& Plotkin, J.B. (2006). Seed dispersal and spatial pattern in tropical trees. Plos Biology, 4, 2132-2137.

37. 
Svenning, J.C. \& Skov, F. (2002). Mesoscale distribution of understorey plants in temperate forest (Kalo, Denmark): the importance of environment and dispersal. Plant Ecology, $160,169-185$.

38.

Uriarte, M., Canham, C.D., Thompson, J., Zimmerman, J.K. \& Brokaw, N. (2005). Seedling recruitment in a hurricane-driven tropical forest: light limitation, density-dependence and 39. the spatial distribution of parent trees. Journal of Ecology, 93, 291-304.

Wiegand, T., Gunatilleke, S. \& Gunatilleke, N. (2007). Species associations in a heterogeneous Sri lankan dipterocarp forest. American Naturalist, 170, E77-E95.

40.

Wiegand, T., Martinez, I. \& Huth, A. (2009). Recruitment in Tropical Tree Species: Revealing Complex Spatial Patterns. American Naturalist, 174, E106-E140.

41.

Wiegand, T. \& Moloney, K.A. (2013). Handbook of spatial point-pattern analysis in ecology. CRC press.

42.

Wright, S.J., Trakhtenbrot, A., Bohrer, G., Detto, M., Katul, G.G., Horvitz, N. et al. (2008). Understanding strategies for seed dispersal by wind under contrasting atmospheric conditions. Proceedings of the National Academy of Sciences of the United States of America, 105, 19084-19089.

Statement of authorship: MK conceptualized the study, all authors planned the study, MK performed the analysis and wrote the first draft of the manuscript and all authors contributed substantially to interpreting results and manuscript revisions.

Data accessibility statement: this work does not use any data. All code used to obtain the results is available in Appendix S4. 